\title{
Analysis of all-optically tunable functionalities in sub-wavelength periodic structures by the Fourier modal method.
}

\author{
Subhajit Beja ${ }^{\mathrm{a}}$,Jani Tervo ${ }^{\mathrm{a}}$, Jorge Francés ${ }^{\mathrm{b}}$, Yuri P. Svirko ${ }^{\mathrm{a}}$, and Jari Turunen ${ }^{\mathrm{a}}$ \\ ${ }^{\text {a} I n s t i t u t e ~ o f ~ P h o t o n i c s, ~ U n i v e r s i t y ~ o f ~ E a s t e r n ~ F i n l a n d, ~ P . O . ~ B o x ~ 111, ~ F I-80101 ~ J o e n s u u, ~}$ \\ Finland

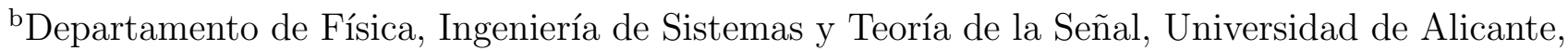 \\ San Vicente del Raspeig Drive, NW, Alicante, Ap. 99, E-3080, Spain
}

\begin{abstract}
We propose the nonlinear Fourier Modal Method (FMM) [J. Opt. Soc. Am. B 31, 2371 (2014)] as a convenient and versatile numerical tool for the design and analysis of grating based next generation all-optical devices. Here, we include several numerical examples where the FMM is used to simulate all-optically tunable functionalities in sub-wavelength periodic structures. At first, we numerically investigate a 1-D periodic nonlinear binary grating with amorphous $\mathrm{TiO}_{2}$. We plot the diffraction efficiency in the transmitted orders against the structure depth for normally incident plane wave. Change in diffraction efficiencies for different incident field amplitudes are evident from the plots. We verify the accuracy of our implementation by comparing our results with the results obtained with the nonlinear Split Field-Finite Difference Time Domain (SF-FDTD) method. Next we repeat the same experiment with vertically standing amorphous Titanium dioxide $\left(\mathrm{TiO}_{2}\right)$ nanowire arrays grown on top of quartz which are periodic in two mutually perpendicular directions and examine the efficiencies in the direct transmitted light for different incident field amplitudes. Our third example includes analysis of a form birefringent linear grating with Kerr medium. With FMM we demonstrate that the birefringence of such a structure can be tuned by all-optical means. As a final example, we design a narrow band Guided Mode Resonance Filter (GMRF). Numerical experiments based on the nonlinear FMM reveal that the spectral tunability of such a filter can be obtained by all-optical means.
\end{abstract}

Keywords: All-optical devices, Kerr-effect, Rigorous modeling, Fourier Modal Method

\section{INTRODUCTION}

In all-optical communication networks, optical data is controlled by signal in optical form and this 'control of light by light' may happen due to the interactions of the optical signal with the nonlinear optical materials chosen to construct components of the communication channel. Third-order optical nonlinearities lead to several interesting physical phenomena such as Optical Kerr effect (OKE) and third-harmonic generation. Nonlinear optical process which results in an intensity dependent refractive index, is known as the optical Kerr effect. This leads to several physical processes with practical interests such as four-wave mixing (FWM) ${ }^{1}$ self-phase modulation $(\mathrm{SPM}),{ }^{2}$ cross-phase modulation $(\mathrm{XPM}),{ }^{3}$ self-focusing and de-focusing, ${ }^{4}$ self-trapping of optical beams, ${ }^{5}$ laser beam breakup,${ }^{6}$ optical phase conjugation, ${ }^{7}$ optical soliton formation, ${ }^{8}$ optical bistability and switching, ${ }^{9,10}$ etc. Over the past few decades, various device applications of Kerr nonlinearity have appeared, especially in integrated optics (IO). ${ }^{11}$ Some of these are power-dependent grating and prism couplers, ${ }^{12-14}$ directional couplers ${ }^{15}$ MachZehnder interferometers, ${ }^{16}$ all-optical switching devices, ${ }^{17,18}$ couplers, flip-flops and logic gates. ${ }^{15,16}$

Many of these nonlinear IO devices employ nanostructures with periodically varying refractive-index profiles (like a diffraction grating); for example; optically tunable filters, multiplexers and demultiplexers, ${ }^{19}$ and distributed-feedback bistable optical devices (DFBS) have been build up. ${ }^{20-22}$ Apart from IO devices, free-space diffractive optical elements with Kerr nonlinear materials have been introduced. ${ }^{23}$ Also, in recent years, there is

Further author information: (Send correspondence to Subhajit Bej.)

Subhajit Bej: E-mail: subhajit.bej@uef.fi, Telephone: +358 408307991

Optical Modelling and Design IV, edited by Frank Wyrowski, John T. Sheridan, Youri Meuret, Proc. of SPIE Vol. 9889, $988906 \cdot$ (C) 2016 SPIE · CCC code: 0277-786X/16/\$18 · doi: 10.1117/12.2227871 
growing research interests in 'Nonlinear metamaterials' and it is already being predicted that these new class of metamaterials might be used to construct parts of the future all-optical communication networks. ${ }^{24}$ However, to accurately model subwavelength periodic diffraction grating like metamaterials with tailored nonlinear optical response, we need advanced numerical simulation tools.

When the dimensions of structural features are of the order of the wavelength of light, rigorous diffraction theory must be used to predict the result both in linear and nonlinear domains. In the linear domain, one may apply space domain methods such as the finite-difference time-domain method (FDTD) ${ }^{25}$ or the finite element method. ${ }^{26}$ Alternatively, spatial-frequency-domain methods, like the differential method ${ }^{27,28}$ and the Fourier modal method (FMM) ${ }^{29,30}$ can also be applied. Some of the rigorous numerical methods mentioned above have been extended for analyzing third-order nonlinear optical phenomena. FDTD and SF-FDTD are efficient, especially if time-dependent phenomena are studied. ${ }^{31-33}$ Further, intrinsically linear methods in the frequency domain can also be used to model third-order optical nonlinearities if an iterative approach is taken. ${ }^{34-37}$

In this paper, we propose the nonlinear $\mathrm{FMM}^{37}$ as a convenient and versatile numerical tool for the design and analysis of grating based next generation free-space all-optical devices that are based on the mechanisms of optical Kerr nonlinearity. This paper is structured in the following manner. In section 2, we include the theoretical framework of our nonlinear calculations based on FMM. Section 3 is devoted to the numerical examples. Finally, discussions are made and conclusions are drawn in section 4.

\section{THEORETICAL BACKGROUND}

In this section, we first describe the optical Kerr effect and then we explain the theoretical framework of the nonlinear $\mathrm{FMM}^{37}$ briefly.

\subsection{Theory of optical Kerr effect (OKE)}

It is a well known fact that the refractive index of many optical materials depends on the intensity of the light used to measure it. When an intense light wave at a frequency $\omega$ propagates through an isotropic third-order nonlinear medium, incoherent light-matter interactions at $\omega$ can be conventionally described in terms of the nonlinear refractive index. ${ }^{38}$ Particularly, if the nonlinear response is instantaneous, the effective refractive index can be calculated using the following equation:

$$
n=n_{0}+n_{2} I_{0} .
$$

Here, $n_{0}$ and $n_{2}$ are the linear and the nonlinear refractive indices of the material medium respectively, and $I_{0}$ is the incident light intensity. However, Eq. (1) holds only for linearly polarized light input and should be modified if one is interested in the effects of the Kerr nonlinearity on the polarization state of the incident elliptically polarized light field. In this case the light wave propagation should be governed by the following constitutive equation:

$$
P_{i}(\omega)=\epsilon_{0}\left[\chi_{i j}^{(1)}(\omega) E_{j}+3 \chi_{i j k l}^{(3)}(\omega=\omega+\omega-\omega) E_{j} E_{k} E_{l}^{*}\right] .
$$

Here, $P_{i}(\omega)$ and $E_{i}(\omega)$ stand for the component of the electric polarization and electric field, respectively, ' $i j k$ ' tag axes of the Cartesian laboratory frame, $\chi_{i j}^{(1)}$ and $\chi_{i j k l}^{(3)}$ are tensors of the first- and third-order optical susceptibilities. These tensors are responsible for the linear and nonlinear refraction, respectively. In an linear isotropic medium, the susceptibility tensor has only diagonal elements, $\chi_{i j}^{(1)}=\left(n_{0}^{2}-1\right) \delta_{i j}$, where $\delta_{i j}$ is the Kronecker delta, while for an isotropic Kerr nonlinear medium $\chi_{i j k l}^{(3)}$ has only two independent components, $\chi_{1122}$ and $\chi_{1221}$. Hence, the material constitutive Eq. (2) in terms of "Effective" linear susceptibility can be written as,

$$
P_{i}(\omega)=\sum_{j} \epsilon_{0} \chi_{i j}^{(\mathrm{eff})} E_{j}
$$

where,

$$
\chi_{i j}^{(\mathrm{eff})}=\left[n_{0}^{2}-1+A|\mathbf{E}|^{2}\right] \delta_{i j}+B \operatorname{Re}\left\{E_{i} E_{j}^{*}\right\}
$$


'Re' denotes the real part and

$$
\begin{aligned}
& A=6 \chi_{1122}-3 \chi_{1221}, \\
& B=6 \chi_{1221} .
\end{aligned}
$$

Equation (4) indicates that in an isotropic third order nonlinear medium, nonlinearity may result in optical anisotropy. Also, it shows that the effective susceptibility is dependent on the polarization state of the incident light.

\subsection{Mathematical framework}

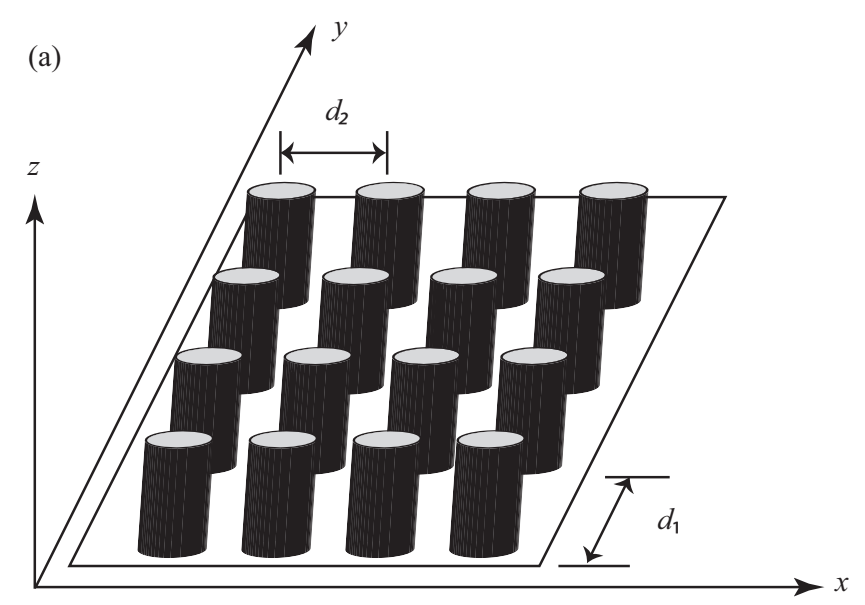

(b)

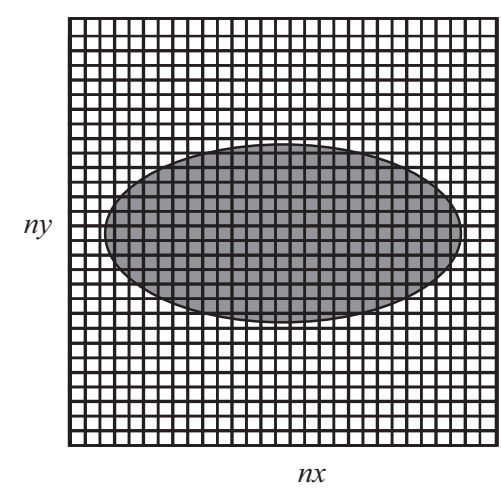

Figure 1. (a) Schematic of the crossed grating. The structure is periodic along $x$ with grating period $d_{1}$ and along $y$ with $d_{2}$. (b) $n_{x} \times n_{y}$ grids of a single layer along $z$. We calculate the field values in each cell using FFT.

Schematic of the crossed grating structure is depicted in Fig. 1(a). $d_{1}$ and $d_{2}$ are the grating periods along $x$ and $y . z$ is the light propagation direction i.e. we consider normal incidence of light from the grating side. We first slice the structure into a number of $z$-independent layers. Then, Maxwell's equations are solved separately in each layer. Lastly, the boundary conditions are used to obtain the final solution. This is best done with the $S$-matrix propagation algorithm. ${ }^{39,40}$ We proceed in a similar fashion but starting with the anisotropic linear Fourier Modal Method (FMM) ${ }^{41}$ which is capable in handling light induced anisotropy. Also in our formulation, the permittivity tensors are field dependent. Our method is an extension of the algorithm introduced by Laine and Friberg for linear gratings composed of isotropic nonlinear media. ${ }^{34}$ In FMM, the electromagnetic fields are expanded into Floquet-Fourier series and medium permittivity (and also permeability) into Fourier series. Then the resulting Maxwell equations are solved as a matrix eigenvalue problem. If monochromatic plane-wave illumination is assumed, any field component in any layer (inside the grating) can be expressed as a superposition of $z$-invariant modes. The essence of our nonlinear FMM is listed below

1. We solve the eigenvalues and the eigenvectors for the grating modes in each layer starting from the linear permittivity, and use the $S$-matrix algorithm ${ }^{39,40}$ to solve the complex field amplitudes of the modes as described in Ref. ${ }^{42}$

2. We solve the electric-field components using the Fast-Fourier Transform (FFT) algorithm in a 3D grid of $n_{x} \times n_{y} \times n_{z}$ points. $n_{x} \times n_{y}$ grid is depicted in Fig. 1(b). $n_{z}$ is the number of thin layers along $z$.

3. Using the solved field components, we calculate the effective linear susceptibility from Eq. (4).

4. We return to the eigenvalue problem, but now using the effective material parameters calculated from the previous step. 
The above described iterative process is continued until we achieve convergence of the results. However, we must note that the change in the field values in two subsequent steps must not be too large otherwise, we will not achieve convergence. Also, we need to use sufficient number of Fourier coefficients to avoid Gibb's phenomenon.

Next, we proceed to reduce the computing time as well as the computing resources needed for the numerical method described above. The detailed mathematical derivations are described in Ref. ${ }^{37}$ Structural symmetries can be used to largely reduce the computation time in FMM because they allow a re-formulation of the eigenvalue problem into a more compact form. ${ }^{43-46}$ We demonstrate that for structures possessing $C_{2 v}$ symmetry we can reduce the computational efforts up to $1 / 8$ th and with TE or TM polarized light up to $1 / 64$ th compared to the most general case. ${ }^{37}$

\section{NUMERICAL RESULTS}

In this section, we include four different numerical examples. In the first example, we design a binary diffraction grating structure with Kerr nonlinear material where the diffraction efficiency can be fine tuned by the intensity of the incident light. Second example includes similar numerical experiment with ALD grown $\mathrm{T}_{\mathrm{i}} \mathrm{O}_{2}$ nanowires. In our third example, we investigate a form birefringent wave plate with Kerr nonlinear medium. For a sufficiently intense light field, the degree of birefringence of such a structure depends on the intensity of the incoming field. Finally, we design a narrow band guided mode resonance transmission filter (GMRF). We show that the spectral response function of the filter can be tuned by varying the incident field intensity.

\section{1 $\mathrm{T}_{\mathrm{i}} \mathrm{O}_{2}$ binary grating}

As a first example, we simulate a 1-D periodic binary grating with amorphous $\mathrm{T}_{\mathrm{i}} \mathrm{O}_{2}$. The substrate material is assumed to be $\mathrm{S}_{\mathrm{i}} \mathrm{O}_{2}$ with $n_{s}=1.46$, the linear refractive index of the pillar material (amorphous $\mathrm{T}_{\mathrm{i}} \mathrm{O}_{2}$ ) is $n_{g}=2$, the nonlinear susceptibility of $\mathrm{T}_{\mathrm{i}} \mathrm{O}_{2}$ is taken to be $\chi^{(3)}=2.1 \times 10^{-20} \mathrm{~m}^{2} / \mathrm{V}^{2}$ ), the vacuum wavelength of the incoming wave $\lambda=633 \mathrm{~nm}$, the grating period $d=2.5 \times \lambda$ and the fill factor $f=0.5$. In Fig. 2(a)-(b), we plot the diffraction efficiencies against the height (in scale of $\lambda$ ) of the pillars assuming that only the pillar material $\left(\mathrm{T}_{\mathrm{i}} \mathrm{O}_{2}\right)$ is nonlinear and ignore the nonlinear coefficients of $\mathrm{S}_{\mathrm{i}} \mathrm{O}_{2}$ and air. This assumption is reasonable as $\mathrm{T}_{\mathrm{i}} \mathrm{O}_{2}$ has 2-3 orders of magnitude larger third-order nonlinear susceptibility. Clearly from the results, the difference between the linear and the nonlinear efficiency curves $\left(I=0.5 \mathrm{MW} / \mu \mathrm{m}^{2}\right)$ increase with increase of the grating depth $h$. From the plots we see that our FMM implementation is in excellent agreement with the SF-FDTD implementation of gratings with Kerr nonlinear media. ${ }^{32}$

\subsection{Arrays of $\mathrm{T}_{\mathrm{i}} \mathrm{O}_{2}$ naowires}

Here, we consider an array of amorphous $\mathrm{T}_{\mathrm{i}} \mathrm{O}_{2}$ (linear refractive index $\mathrm{n}_{\mathrm{g}}=2$ and third-order susceptibility $\left.\chi^{(3)}=1.47 \times 10^{-20} \mathrm{~m}^{2} / \mathrm{V}^{2}\right)$ nanowires. The nanowires are cylindrical shaped and the height/diameter aspect ratio of the wires $h / D$ is $\sim 10$. The geometry of the structure is depicted in Fig. 3(a). The nanowires are grown on top of quartz by the atomic layer deposition (ALD). In our numerical experiment, we assume a plane wave of wavelength $\lambda=532 \mathrm{~nm}$ to be normally incident on the structure from air. In Fig. 3(b), we plot the diffraction efficiency against the height (in scale of $\lambda$ ) of the wires. The black solid curve is the linear case where the electric field intensity of the input plane wave is not sufficient to generate Kerr nonlinearity inside the wires. The red dotted curve is for the incident electric field intensity $\mathrm{I}=50 \mathrm{GW} / \mathrm{cm}^{2}$. From the plots we observe that though the effect of Kerr nonlinearity is rather small, with growing thickness of the nanowires, the difference between the linear and the nonlinear efficiency curves becomes more and more prominent. Hence, by employing very long wires, it is possible to tune the diffraction efficiency by all-optical means.

\subsection{Form birefringent wave plate}

Dielectric subwavelength diffraction gratings with grating period $\Lambda \leq \lambda / 10$ yield strong birefringence in direct transmission due to their structural anisotropy. This type of birefringence is termed as 'Form birefringence'. ${ }^{47}$ Form birefringent gratings have wide commercial applications especially in constructing devices such as wave plates, retarders, polarizing beam splitters etc. ${ }^{48-51}$ Though birefringent gratings with linear materials have been studied extensively in the past, form birefringence of gratings with Kerr media came to research focus only very recently. ${ }^{52}$ Here, we design a subwavelength form birefringent grating structure with 1-D periodicity. 


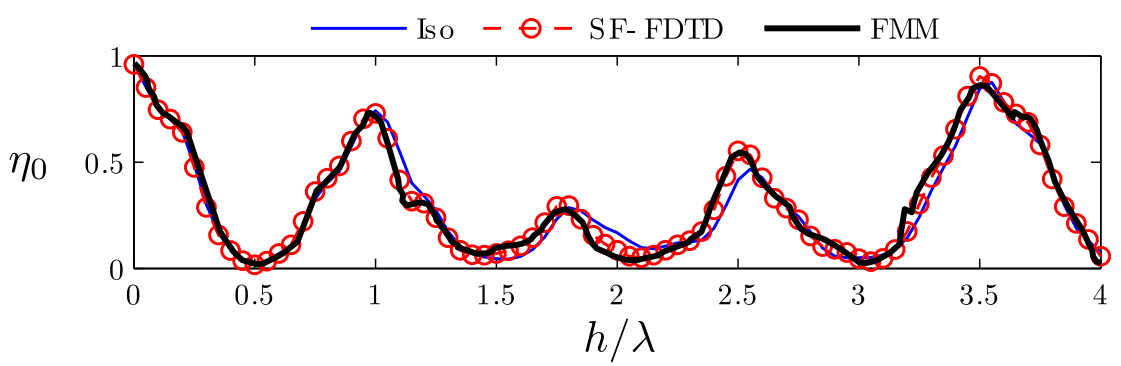

(b)

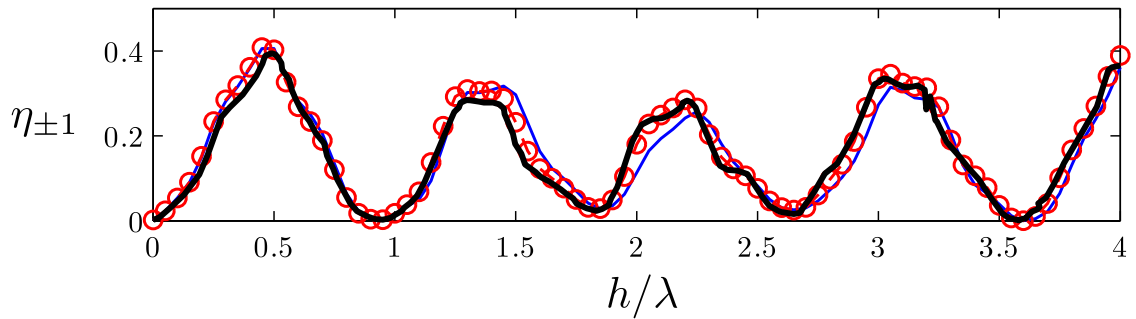

Figure 2. Diffraction efficiency curves of (a) direct transmitted order and (b) transmitted first-orders. Red circles are SF-FDTD results and it is clear from the plots that these red circles fit very well with the results obtained by the nonlinear FMM (solid black lines). Blue solid line is the linear curve.

The wavelength of the incident light is $\lambda_{0}=532 \mathrm{~nm}$, grating fill factor $=0.5$, and $\Lambda=50 \mathrm{~nm}$. The grating material is chosen to be $\mathrm{S}_{\mathrm{i}} \mathrm{O}_{2}$ with linear refractive index $n_{\mathrm{g}}=1.47$, the grooves are assumed to be filled with DDMEBT polymer with $\chi^{(3)}=1.19 \times 10^{-17} \mathrm{~m}^{2} / \mathrm{V}^{2}$, negligible two-photon absorption (TPA) and linear refractive index $n_{\mathrm{s}}=1.88$. The substrate is $\mathrm{S}_{\mathrm{i}} \mathrm{O}_{2}$. Plane wave is assumed to be incident normally from air. In Fig. 4(a) we plot the birefringence viz. the phase difference between $x$ and $y$ components $\left(\Phi_{t x 00}\right.$ and $\left.\Phi_{t y 00}\right)$ of the direct transmitted light both for the linear and the nonlinear $\left(\mathrm{I}=10 \mathrm{GW} / \mathrm{cm}^{2}\right)$ cases. From the plots it is evident that by choosing the incident field intensity, the grating material, the surrounding medium and the grating dimensions carefully, it is possible to achieve wave retardation $\sim 10^{\circ}$ s solely by optical means. In Fig. $4(\mathrm{~b})$, the nonlinear form birefringence (the difference between the linear and the nonlinear cases) is plotted as a function of the grating depth $h$ for 4 different polarization states of the incident electric field. It is evident from the plots that the strongest effect is observed with an incident field that is linearly polarized in the $y$ direction, and the weakest with the $x$ polarized field.

\subsection{Guided Mode Resonance Filter}

Waveguide grating in its most basic configuration includes a relief grating layer, a waveguide layer and a substrate layer. Depending on the structural dimensions and the mounting conditions, strong resonance phenomenon can be observed. At resonance, a guided mode is excited by the incident wave. This guided mode interferes with the direct transmitted/reflected wave destructively and as a result 100 percent efficiency can be achieved in direct reflection/transmission. Also, at resonance there is large field confinement inside the structure. This enhances the effective nonlinearity inside the structure. Here we design a $\mathrm{Si}_{3} \mathrm{~N}_{4}$ (Silicon Nitride) GMRF on top of quartz which acts as a narrow-band reflector. The structure is assumed to be periodic in $x$ direction with grating period $d=930 \mathrm{~nm}$, fill factor is assumed to be $f=0.5$. We assume plane wave propagation along $z$ from the grating side as depicted in Fig. 5(a). The nonlinear susceptibility is taken to be $\chi^{(3)}=3 \times 10^{-20} \mathrm{~m}^{2} / \mathrm{V}^{2}$, TPA of $\mathrm{Si}_{3} \mathrm{~N}_{4}$ is assumed to be negligible and $A=2 B$ is used in Equation (4). Thickness of the grating layer is $\delta=20 \mathrm{~nm}$ and that of the waveguide layer is $t=380 \mathrm{~nm}$. From Fig. 5(b) we observe shift of the reflection peak towards the longer wavelengths as the intensity of the incident plane wave increases. Hence, from 100 percent transmission to 100 percent reflection can be achieved with this kind of structure keeping the wavelength fixed but varying the incident field intensity. This kind of structure may be employed to construct all-optically tunable filters. 
(a)
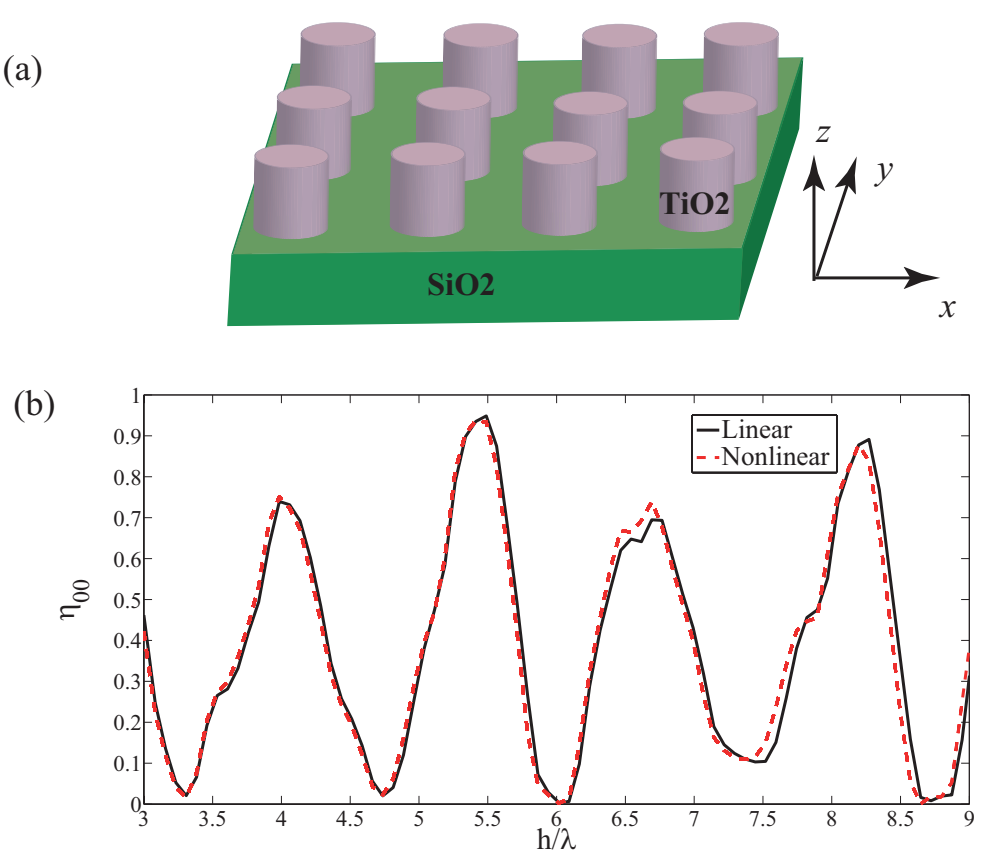

Figure 3. (a) Schematic of the nanowires. The structure is periodic along both $x$ and $y$ directions with periodicity $d_{1}=d_{2}=0.9 \times \lambda$. Diameter of the wires $=0.6 \times d_{1}$. The wires are grown by ALD on top of quartz substrate. (b) Efficiency in direct transmission. The black solid curve is plotted by assuming the incident field intensity extremely low i.e. the linear case. The red dotted curve is plotted assuming the incident field intensity $\mathrm{I}=5 \times 10^{14} \mathrm{~W} / \mathrm{m}^{2}$. Clearly from the plots, the difference between the linear and the nonlinear curves increase with the growing thickness of the wires.

(a)

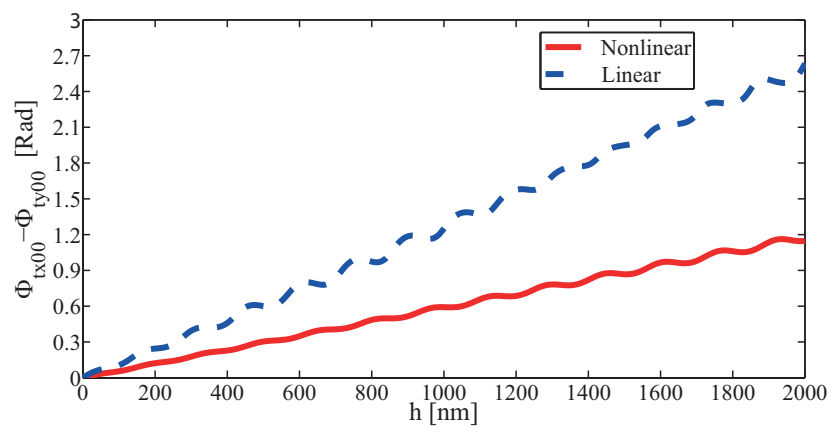

(b)

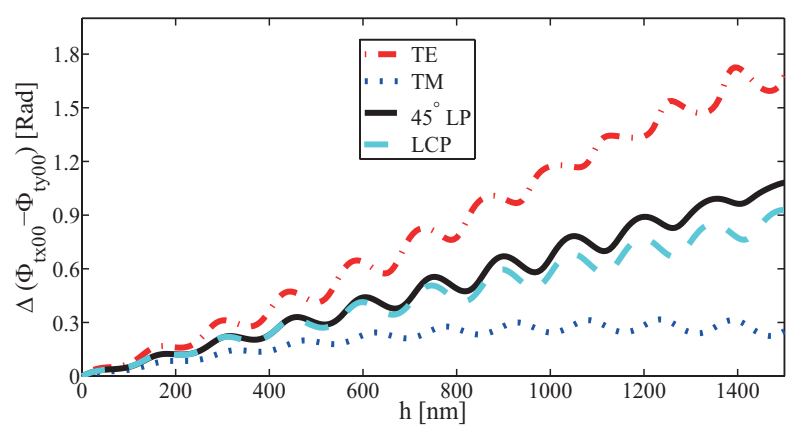

Figure 4. (a) Birefringence viz. the phase difference $\left(\Phi_{t x 00}-\Phi_{t y 00}\right)$ between the $x$ and $y$ components of the direct transmitted field. $45^{\circ}$ linearly polarized field is assumed to be normally incident on the structure from air. (b) the nonlinear birefringence viz. the difference between the linear and the nonlinear results for different polarization states of the incident electric field. The strongest effect is observed with $y$ polarized field while the weakest effect with $x$ polarized field.

\section{DISCUSSIONS AND CONCLUSIONS}

Devices whose properties can be tuned by all-optical means are already being investigated thoroughly for suitable commercial use. Very recently, a new class of electromagnetic metadevices known as tunable metadevices, where the device properties can be tuned by all-optical means have emerged. It is already being predicted that in near future, these new class of metamaterials will find significant applications in relation to optical communication. Hence, new fabrication and experimental schemes need to be assisted by advanced numerical simulation techniques for some known standard nonlinear processes where we must use the full-wave numerical 
(a)

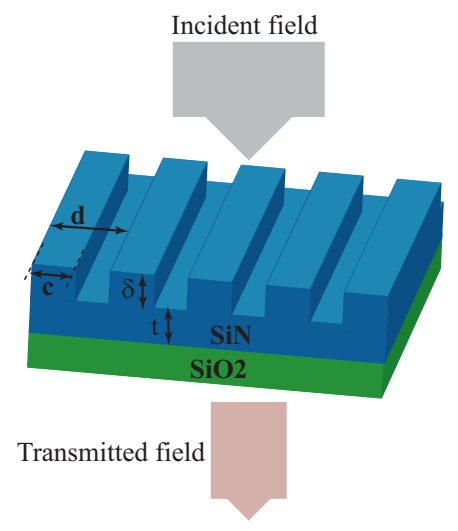

(b)

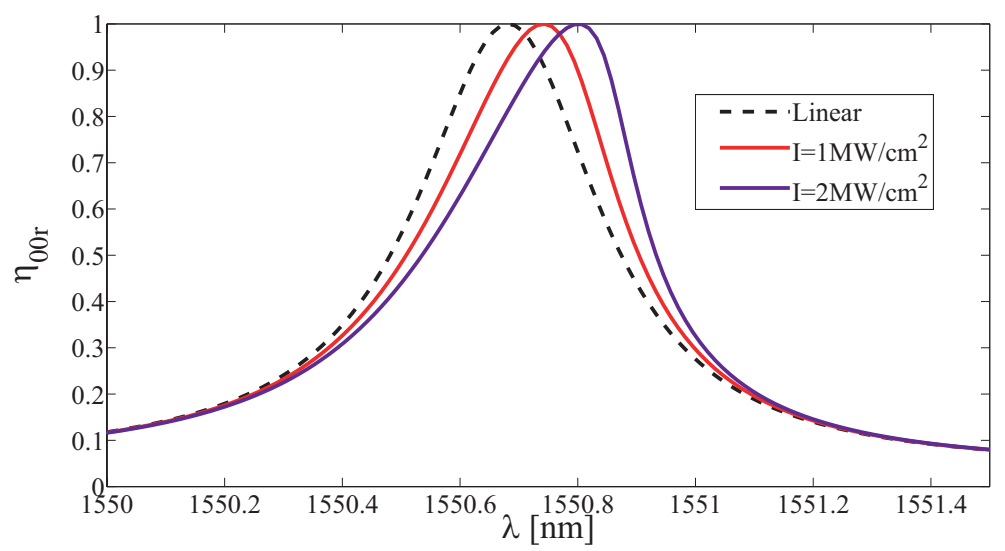

Figure 5. (a) Geometry of the GMRF. Thickness of the grating layer $\delta=20 \mathrm{~nm}$, and thickness of the waveguide layer $t=380 \mathrm{~nm}$. (b) Efficiency of 0th reflected order. With growing intensity of the incident field, we observe shift of the reflectance curve towards longer wavelengths.

simulation approach starting from the Maxwell's equations.

Our nonlinear FMM is a good candidate for designing all-optically tunable functionalities in the context of periodic nonlinear metamaterials. However, there are some limitations. For materials with high TPA coefficients, this method can not be used because besides nonlinear refraction we must also take into account nonlinear absorption. Also, in our numerical implementation only monochromatic plane wave incidence is assumed. We aim to overcome these issues in our future works so that the method can also be applied seamlessly in the context of ultrafast nonlinear optics and also with materials with high TPA.

\section{ACKNOWLEDGMENTS}

This work is partially supported by the Academy of Finland (contract 285880).

\section{REFERENCES}

[1] Abrams, R. L. and Lind, R. C., "Degenerate four-wave mixing in absorbing media," Opt. Lett. 2, 94-96 (1978).

[2] Alfano, R. R. and Shapiro, S. L., "Observation of self-phase modulation and small-scale filaments in crystals and glasses," Phys. Rev. Lett. 24, 592-594 (1970).

[3] Agrawal, G. P., "Modulation instability induced by cross-phase modulation," Phys. Rev. Lett. 59, 880-883 (1987).

[4] Metropolis, N., Rosenbluth, A. W., Rosenbluth, M. N., Teller, A. H., and Teller, E., "Self-focusing of optical beams," Opt. Lett. 2, 94-96 (1978).

[5] Chiao, R. Y., Garmire, E., and Townes, C. H., "Self-trapping of optical beams," Phys. Rev. Lett. 13, 479-482 (1964).

[6] Chilingarian, Y. S., "Self-focusing of inhomogeneous laser beams and its effect on stimulated scattering," JETP Lett. 28, 832-835 (1969).

[7] Zel'Dovich, B., Pilipetsky, N., and Shkunov, V., [Principles of Phase Conjugation], Springer-Verlag, Berlin (1985).

[8] Mollenauer, L. F., Stolen, R. H., and Gordon, J. P., "Experimental observation of picosecond pulse narrowing and solitons in optical fibers," Phys. Rev. Lett. 45, 1095-1098 (1980). 
[9] Szőke, A., Daneu, V., Goldhar, J., and Kurnit, N. A., "Bistable optical element and its applications," Appl. Phys. Lett. 15, 376-378 (1969).

[10] Gibbs, H. M., McCall, S. L., and Venkatesan, T. N. C., "Differential gain and bistability using a sodium-filled fabry-perot interferometer," Phys. Rev. Lett. 36, 1135-1138 (1976).

[11] Stegeman, G. I., Wright, E. M., Finlayson, N., Zanoni, R., and Seaton, C. T., "Third order nonlinear integrated optics," J. Lightwave Technol. 6, 953-970 (1988).

[12] Fortenberry, R. M., Moshrefzadeh, R., Assanto, G., Mai, X., Wright, E. M., Seaton, C. T., and Stegeman, G. I., "Power-dependent coupling and switching in prism and grating coupling to zno waveguides," Appl. Phys. Lett. 49, 687-689 (1986).

[13] Pardo, F., Chelli, H., Koster, A., Paraire, N., and Laval, S., "Experimental and theoretical study of ultrafast optical switching using guided mode excitation in silicon on sapphire," IEEE J. Quantum Electron. 23, 545550 (1987).

[14] Assanto, G., Svensson, B., Kuchibhatla, D., Gibson, U. J., Seaton, C. T., and Stegeman, G. I., "Prism coupling in zns waveguides: a classical example of a nonlinear coupler," Opt. Lett. 11, 644-646 (1986).

[15] Jensen, S. M., "The nonlinear coherent coupler," IEEE J. Quantum Electron. 18, 1580-1583 (1982).

[16] Lattes, A., Haus, H., Leonberger, F. J., and Ippen, E. P., "An ultrafast all-optical gate," IEEE J. Quantum Electron. 19, 1718-1723 (1983).

[17] Radic, S., George, N., and Agrawal, G. P., "Optical switching in $\lambda / 4$ shifted nonlinear periodic structures," Opt. Lett. 19, 1789-1791 (1994).

[18] Radic, S., George, N., and Agrawal, G. P., "Theory of low-threshold optical switching in nonlinear phaseshifted periodic structures," J. Opt. Soc. Am. B 12, 671-680 (1995).

[19] Wang, Y., Wei, Y., Huang, Y., Tu, Y., Ng, D., Lee, C., Zheng, Y., Liu, B., and Ho, S. T., "Silicon/iii- v laser with super-compact diffraction grating for wdm applications in electronic-photonic integrated circuits," Opt. Express 19, 2006-2013 (2011).

[20] Winful, H. G. and Stegeman, G. I., "Applications of nonlinear periodic structures in guided wave optics," Proc. SPIE 517, 214-218 (1984).

[21] Vincent, P., Paraire, N., Nevière, M., Koster, A., and Reinisch, R., "Gratings in nonlinear optics and optical bistability," J. Opt. Soc. Am. B 2, 1106-1116 (1985).

[22] Hegde, R. S. and Winful, H. G., "Optical bistability in periodic nonlinear structures containing left handed materials," Microw. Opt. Technol. Lett. 46, 528-530 (2005).

[23] Manela, O. and Segev, M., "Nonlinear diffractive optical elements," Opt. Express 15, 10863-10868 (2007).

[24] Lapine, M., Shadrivov, I. V., and Kivshar, Y. S., "Colloquium: Nonlinear metamaterials," Rev. Mod. Phys. 86, 1093-1123 (2014).

[25] Kunz, K. S. and Luebbers, R. J., [The Finite Difference Time Domain Method for Electromagnetics], CRC Press, New York (1993).

[26] Jin, J. M., [The Finite Element Method in Electromagnetics], Wiley-IEEE, New Jersey (2002).

[27] Nevière, M. and Popov, E., [Light Propagation in Periodic Media: Differential Theory and Design], Marcel Dekker, New York (2002).

[28] Popov, E. and Nevière, M., "Maxwell equations in fourier space: fast-converging formulation for diffraction by arbitrary shaped, periodic, anisotropic media," J. Opt. Soc. Am. A 18, 2886-2894 (2001).

[29] Li, L., "New formulation of the fourier modal method for crossed surface-relief gratings," J. Opt. Soc. Am. A 14, 2758-2767 (1997).

[30] Kim, H., Park, J., and Lee, B., [Fourier Modal Method and Its Applications in Computational Nanophoton$i c s]$, CRC Press, New York (2012).

[31] Soljačič, M. and Joannopoulos, J. D., "Enhancement of nonlinear effects using photonic crystals," Nat. Mater. 3, 211-219 (2004).

[32] Francés, J., Tervo, J., and Neipp, C., "Split-field finite-difference time-domain scheme for kerr-type nonlinear periodic media," Prog. Electromagn. Res. 134, 559-579 (2013).

[33] Francés, J., Tervo, J., and Márquez, A., "Tensorial split-field finitedifference time-domain approach for second- and third-order nonlinear materials," J. Opt. Soc. Am. B 30, 1711-1719 (2013). 
[34] Laine, T. A. and Friberg, A. T., "Rigorous volume grating solution to distortion correction in nonlinear layered media near a phase-conjugate mirror," Opt. Commun. 159, 93-98 (1999).

[35] Bonod, N., Popov, E., and Nevière, M., "Fourier factorization of nonlinear maxwell equations in periodic media: application to the optical kerr effect," Opt. Commun. 244, 389-398 (2005).

[36] Bonnefois, J. J., Guida, G., Priou, A., Nevière, M., and Popov, E., "Simulation of two-dimensional kerr photonic crystals via fast fourier factorization," J. Opt. Soc. Am. 23, 842-847 (2006).

[37] Bej, S., Tervo, J., Svirko, Y. P., and Turunen, J., "Modeling the optical kerr effect in periodic structures by the linear fourier modal method," J. Opt. Soc. Am. B 31, 2371-2378 (2014).

[38] Boyd, R. W., [Nonlinear Optics], Academic Press, New York (2003).

[39] Li, L., "Formulation and comparison of two recursive matrix algorithms for modeling layered diffraction gratings," J. Opt. Soc. Am. A 13, 1024-1035 (1996).

[40] Li, L., "Formulation and comparison of two recursive matrix algorithms for modeling layered diffraction gratings," J. Opt. Soc. Am. A 20, 650-660 (2003).

[41] Li, L., "Fourier modal method for crossed anisotropic gratings with arbitrary permittivity and permeability tensors," Journal of Optics A: Pure and Applied Optics 5, 345-355 (2003).

[42] Vallius, T., Tervo, J., Vahimaa, P., and Turunen, J., "Electromagnetic field computation in semiconductor laser resonators," J. Opt. Soc. Am. A 23, 906-911 (2006).

[43] Zhou, C. and Li, L., "Formulation of the fourier modal method for symmetric crossed gratings in symmetric mountings," Journal of Optics A: Pure and Applied Optics 6, 43-50 (2004).

[44] Bai, B. and Li, L., "Reduction of computation time for crossed-grating problems: a group-theoretic approach," J. Opt. Soc. Am. A 21, 1886-1894 (2004).

[45] Bai, B. and Li, L., "Group-theoretic approach to enhancing the fourier modal method for crossed gratings with one or two reflection symmetries," Journal of Optics A: Pure and Applied Optics 7, 271-278 (2005).

[46] Bai, B. and Li, L., "Group-theoretic approach to enhancing the fourier modal method for crossed gratings of plane group p3," J. Mod. Opt. 52, 1619-1634 (2005).

[47] Born, M. and Wolf, E., [Principles of Optics, Chap. XV], Cambridge University Press, Cambridge, 7th ed. (1999).

[48] Flanders, D. C., "Submicrometer periodicity gratings as artificial anisotropic dielectrics," Appl. Phys. Lett. 42, 492-494 (1983).

[49] Richter, I., Sun, P. C., Xu, F., and Fainman, Y., "Design considerations of form birefringent microstructures," Appl. Opt. 34, 2421-2429 (1995).

[50] Kikuta, H., Yoshida, H., and Iwata, K., "Ability and limitation of effective medium theory for subwavelength gratings," Opt. Rev. 2, 92-99 (1995).

[51] Gu, C. and Yeh, P., "Design considerations of form birefringent microstructures," Opt. Lett. 21, 504-506 (1996).

[52] Bej, S., Tervo, J., Svirko, Y. P., and Turunen, J., "Form birefringence in kerr media: analytical formulation and rigorous theory," Opt. Lett. 40, 2913-2916 (2015). 